\section{Macro- and microbehavioral response to viscosity among rats licking saccharin}

\author{
W. GREGG WILCOVE and JAMES ALLISON \\ Indiana University, Bloomington, Ind. 47401
}

Effects of viscosity upon licking were studied by providing rats with thick and thin $.4 \%$ saccharin solutions in separate 1 -h sessions. Volumetric intake/lick was greater for the thin than for the thick solution and was the only measure affected by viscosity. As the session progressed, the two solutions caused (1) comparable satiation effects, as evidenced by comparable decreases in several indices of the average rate of ingestion; (2) comparable decreases in the average duration of the lick; and (3) no reliable change in the average volumetric intake of the lick. The results were related to earlier comparisons of liquid food and saccharin and indicated that the differences produced by the latter solutions are not fully attributable to their differential viscosity.

The rat's licking behavior is capable of numerous adjustments which depend upon the properties of the solution being licked (see Corbit \& Luschei, 1969; Hulse, 1966; Hulse \& Suter, 1968, 1970; Petrie \& Hulse, 1971). Allison (1971) compared a relatively viscous liquid food (Nutrament) with a less viscous, nonnutritive saccharin solution during a 60-min test session. Saccharin licking resulted in several "macrobehavioral" indications of satiation, such as a steady increase in the time required to complete successive blocks of 500 licks. In contrast, Nutrament licking showed no evidence of satiation. Differences were also evident at a "microbehavioral" level. For example, saccharin caused no change in the average duration of the lick (onset to offset), confirming Hulse (1967), whereas Nutrament caused a decrease in lick duration as the session progressed, confirming Allison \& Castellan (1970). Furthermore, rats took in a greater volume/lick drinking saccharin than when drinking Nutrament.

The source of these behavioral differences is not yet clear, as the two solutions differed on several dimensions, including flavor, caloric content, and viscosity. The work reported here attempted to evaluate the role of viscosity alone by comparing two $.4 \%$ Na-saccharin solutions: a normal "thin" solution and a "thick" solution which approximated the viscosity of Nutrament.

\section{METHOD}

Ss were six male albino rats about 1 year old, obtained from the Holtzman Company (Madison, Wisconsin). They were experimentally naive and had been maintained on a 12-h day-night cycle with ad lib food and water. Two weeks prior to, and for the duration of the experiments, Ss were fed and watered $1 \mathrm{~h}$ a day at the end of each session.
The apparatus has been described in detail elsewhere (Allison, 1971). It consisted of a 20-cm-square $x$ 14-cm-high box with a $1.5-\mathrm{cm}$-diam hole in a side wall, about $5 \mathrm{~cm}$ from the front door wall and about $4 \mathrm{~cm}$ off the floor. Affixed to a rigid stand outside the box, a $50-\mathrm{ml}$ burette graduated in $.1-\mathrm{ml}$ divisions was fitted with a glass drinking spout, with an aperture of $3 \mathrm{~mm}$, and connected to a BRS drinkometer. The tip of the drinking spout was recessed $7 \mathrm{~mm}$ from the inside wall of the experimental chamber, and access to the tube was controlled automatically by a sliding bar on the outside of the chamber which opened or closed the hole in the experimental chamber.

On Pretest Days 1, 2, and 3 all Ss were placed in the apparatus for $1 \mathrm{~h}$ and the tube filled with water was exposed. Following each block of 250 licks the hole was automatically closed. At this time the $\mathrm{E}$ entered the experimental room, recorded the volume drunk to the nearest $.1 \mathrm{ml}$, and reexposed the tube approximately 30 sec later. Total time in contact with the tube (cumulative duration of 250 licks) was recorded to the nearest millisecond. Also recorded was the latency of the last lick in the block. The number of licks in the block was verified by an electromechanical counter which functioned independently of the lick counter in the control circuit. The same procedure was followed during the test session.

Those Ss failing to reach a criterion of at least eight complete blocks on a tube during Day 3 were placed on $46 \mathrm{~h}$ water deprivation and received an extra pretest session on Day 4.

Following this procedure, Ss received two 1 -h test sessions separated by 3 days. The "thin" test solution consisted of $0.4 \%$ $\mathrm{Na}-\mathrm{Saccharin}(\mathrm{w} / \mathrm{v})$ in distilled water. Its viscosity was 1.0718 centipoise, as compared with 1.0050 centipoise for water. "Thick" $.4 \%$ saccharin solution was produced by adding $6.5 \%(\mathrm{w} / \mathrm{v})$ polyethylene glycol compound 20-M (Union Carbide Chemicals)-a colorless, flavorless, odorless compound-to the $0.4 \%$ saccharin solution. The resulting viscosity of 7.0714 centipoise was comparable to that of vanilla Nutrament, 8.0391.

Half of the Ss received the thick solution in the first test session, and the thin in the second. The other half received thin followed by thick.

\section{RESULTS}

Each S's record was vincentized into four quarters, each containing approximately $1 / 4$ of the 250-lick blocks. Within-session changes were evaluated by one-way analyses of variance $(\mathrm{df}=3,15)$ followed by trend tests $(\mathrm{df}=1,15)$.

Macrobehavioral Measures

Three measures reflecting rate of licking, rate of intake, and rate of contact with the tube were derived from the time required to complete the block of licks. A decrease in any of these measures across quarters would indicate satiation. Each measure showed that the two solutions caused significant and comparable amounts of satiation as the session progressed.

Licks per second is the number of licks in a block divided by the amount of time required to complete the block of licks, and milliliters per second is defined similarly. Each solution caused a linear decrease in licks per second across quarters $(p<.001$ in both cases), as well as milliliters per second $(p<.05$ in both cases $)$.

Milliseconds per second is the proportion of time in contact with the tube, obtained by dividing cumulative lick duration for the block by the time required to complete the block. Each solution caused a linear decrease in this measure ( $p<.001$ in both cases). The trend caused by the thin solution also contained a significant quadratic component $(p<.001)$.

These three macrobehavioral measures appear at the right of Fig. 1. Statistical analyses revealed no difference between the trends produced by the two solutions. The functions shown in Fig. 1 may suggest that the thin solution caused more pronounced decreases than the thick, but analyses of variance revealed no significant interactions between solution and quarter (all ps $>.05$ ).

Both solutions reproduced the trends which Allison (1971) reported for normal .4\% saccharin rather than Nutrament. It follows that the macrobehavioral differences between normal saccharin and Nutrament can not be attributed to differential viscosity. Thick $.4 \%$ saccharin which approximates the viscosity of Nutrament produces a satiation effect 

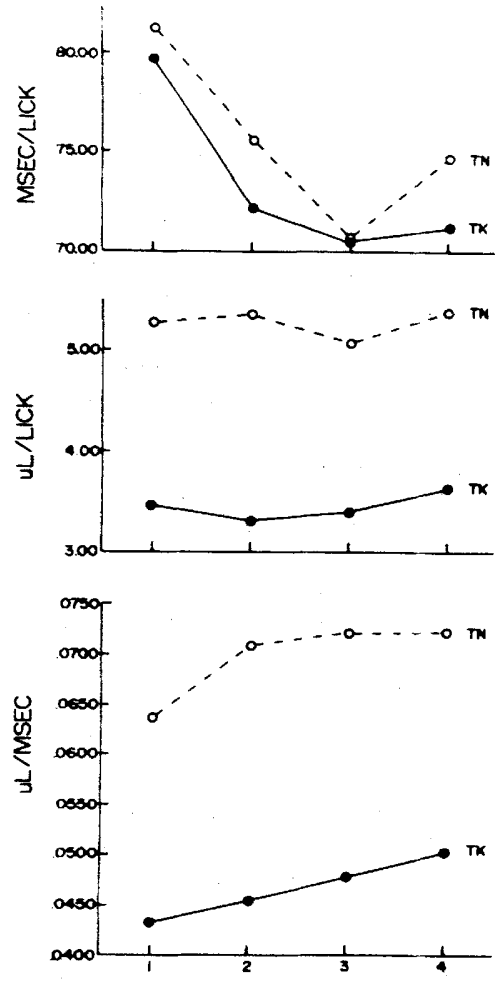

QUARTERS
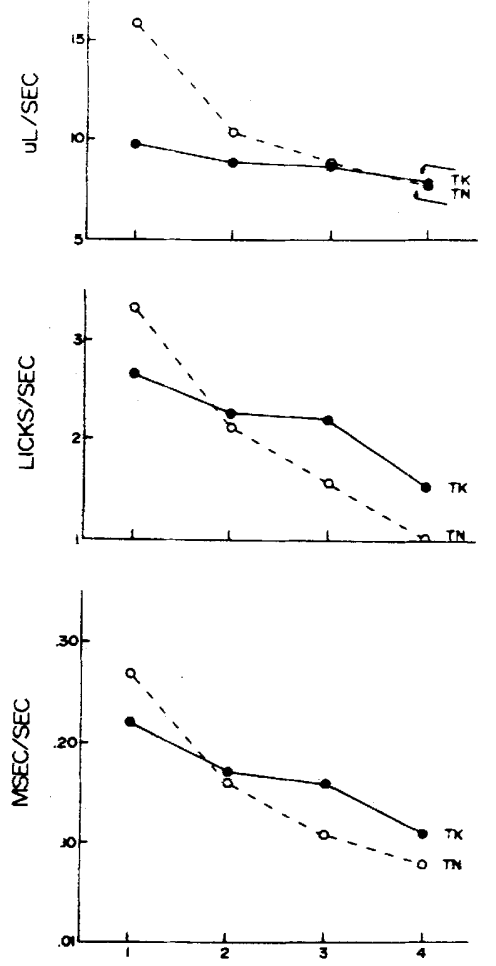

QUARTERS

Fig. 1. Microbehavioral (left) and macrobehavioral (right) ingestion patterns of rats $(N=6)$. Each data point consists of at least 3,000 licks as all six functions consist of vincentized quarters of a session. The three microbehavioral functions depict rat ingestive behavior at the level of each lick. The macrobehavioral graphs are functions of time spent in the session.

which is not distinguishable from the effect of thin .4\% saccharin. Some other variable, such as flavor or caloric content, must be responsible for the ability of Nutrament to maintain a higher rate of ingestion than saccharin during a 60-min test session.

Microbehavioral Measures

Milliseconds per lick is the total time in contact with the tube divided by the number of licks in the block. The thick solution caused a significant decrease in milliseconds per lick, reproducing the effect of high-viscosity liquid food (Allison \& Castellan, 1970; Allison, 1971). The trend had significant linear and quadratic components $(p<.001$, $\mathrm{p}<.01)$. The thin solution caused precisely the same trend as the thick, in contrast with earlier investigations in which regular saccharin solution failed to cause any reliable change in milliseconds per lick (Allison, 1971; Hulse, 1967).

The volumetric measure, microliters per lick, is the total volumetric intake divided by the number of licks in the block. Averaged across the session as a whole, microliters per lick was significantly greater for the thin than for the thick solution $(p<.001)$, regulation of ingestion. As expected, the high-viscosity saccharin produced a lower volume/lick and volume/millisecond score than ordinary saccharin.

However, viscosity is not the only variable operative in rat licking behavior. Viscosity did not affect lick duration, and the thick solution failed to reproduce the macrobehavioral functions obtained for Nutrament under comparable experimental conditions (Allison, 1971). Therefore, the macrobehavioral differences between Nutrament and saccharin are not attributable to viscosity. Furthermore, the thin saccharin curves obtained by Allison (1971) and Hulse (1967) differed from our experiment as the present animals decreased their time-on-tube per lick within the session while their animals showed no change.

Time spent in the apparatus may be the reason for the present study's failure to replicate ordinary saccharin functions found by previous investigators. Hulse (1967) and Allison (1971) used Ss which had been exposed to an experimental regimen extensively prior to testing while the Ss of the present study were exposed to the apparatus a maximum of four times, and usually only three. (Allison's animals drank for 480 training minutes prior to test and Hulse's Ss were trained for 20,000 licks or $200 \mathrm{~min}$ ). In fact, preliminary data from this laboratory indicate that practice has a large effect on both the shape and the elevation of all microbehavioral and macrobehavioral functions. The results of the present study also demonstrate that there is no correspondence between lick duration and lick intake (although high viscosity is associated with low volumetric intake/lick). Allison (1971) showed that rats drinking Nutrament decrease lick duration within the session while volume per lick increased. Rats drinking saccharin showed no change in the duration of each lick, but volume per lick increased and then decreased. The present study found a general decrease in lick duration while there was no change within the session in microliters/lick.

The results of the present study lend further support to and extend the findings of previous investigations by confirming that micro- and macrobehavioral measures do not necessarily covary. While a $S$ may show macrobehavioral satiation on a fluid, the duration of each lick and volume per lick may increase, decrease, or stay the same. In this experiment, rats satiating on ordinary saccharin showed a decrease in lick duration within the session, while 
other investigators have reported satiation on saccharin with no alterations in milliseconds/lick. The present experiment showed that under some conditions, rats will hold microliters/lick constant, while other experiments have indicated that this measure may increase or decrease, independently of macrobehavioral satiation.

Although some ingestive regulation seems due to the physical parameters of the situation, other aspects of licking in rats seem to be controlled by as yet unspecified processes. Those processes controlling microbehavioral ingestion patterns are probably orthogonal to the macrobehavioral processes accompanying it.

\section{REFERENCES}

ALLISON, J. Microbehavioral features of nutritive and non-nutritive drinking in the rat. Journal of Comparative \& Physiological Psychology, 1971, 76 , 408-417.

ALLISON, J., \& CASTELLAN, N. J. Temporal characteristics of nutritive drinking in rats and humans. Journal of Comparative \& Physiological Psychology, 1970, 70, 116-125.

CORBIT, J., \& LUSCHEI, E. Invariance of the rat's rate of drinking. Journal of Comparative \& Physiological Psychology, 1969, 69, 119-125.
HULSE, S, Stimulus intensity and magnitude of the licking reflex. Psychonomic Science, 1966, 6, 33-34.

HULSE, S. Licking behavior in relation to saccharin concentration and shifts in fixed ratio reinforcement. Journal of Comparative \& Physiological Psychology, $1967,64,478-484$.

HULSE, S., \& SUTER, S. 1 drop licking in rats. Journal of Comparative \& Physiological Psychology, 1968, 66. 536-539.

HULSE, S., \& SUTER, S. Emitted and elicited behavior: An analysis of some learning mechanisms associated with fluid intake in rats. Learning \& Motivation, 1970, 1, 304-315.

PETRIE, H. L., \& HULSE, S. DRL effects on licking behavior of rats. Paper presented to Midwestem Psychological Association, 1971. 